\title{
Immunohistochemical characterization of stem cell and differentiation markers of the dental pulp of human natal teeth
}

\author{
Heeresh Shetty ${ }^{1}$, Adesh Kakade², Shishir Shetty ${ }^{3}$, Prasanna Neelakantan ${ }^{4}$, Saurabh Nagar ${ }^{5}$, \\ Rajiv S Desai ${ }^{5}$ \& Kavita Beri*,6,7 \\ ${ }^{1}$ Department of Conservative Dentistry \& Endodontics Nair Hospital Dental College, Mumbai, India \\ ${ }^{2}$ Department of Pediatric Dentistry, Nair Hospital Dental College, Mumbai, India \\ ${ }^{3}$ Department of Conservative Dentistry \& Endodontics, A.B Shetty Institute of Dental Sciences, Mangalore, India \\ ${ }^{4}$ Discipline of Endodontology, Faculty of Dentistry, University of Hong Kong, Hong Kong \\ ${ }^{5}$ Department of Oral \& Maxillofacial Pathology, Nair Hospital Dental College, Mumbai, India \\ ${ }^{6}$ Biomedical Engineering, Rutgers University of New Jersey, New Brunswick, NJ, 08854, USA \\ ${ }^{7}$ Center for Dermal Research, NJ Center for Biomaterials Rutgers, The State University of New Jersey, NJ, 07302, USA \\ *Author for correspondence: beri.kavita@gmail.com
}

\begin{abstract}
Aim: Dental pulp stem cells, which are primarily derived from the pulp tissues of human teeth, have rarely been obtained from natal teeth. This study investigated the stem cell and differentiation markers of the dental pulp of natal teeth using immunohistochemistry. Materials \& methods: The pulp tissue from extracted natal teeth $(n=2)$ of a 20 -day-old healthy male was examined for immunohistochemical expression of stem cell (Oct-4 and SOX 2) and differentiation markers (Nestin, CD 44, desmin, osteopontin and Ki67). Results: The pulp tissue of the natal teeth expressed immunopositivity for nestin, CD 44 and SOX2. Conclusion: Natal teeth, if preserved properly, could serve as sources of dental pulp stem cells that are an improvement on deciduous teeth.
\end{abstract}

Lay abstract: Natal teeth are present at birth. They are usually removed prophylactically to avoid the complications such as aspiration, ulcerations on the tongue and injuries to the mother during feeding. To study discarded natal teeth as a potential stem cell source, immunohistochemistry was used to check for the expression of stem cells and markers on the dental pulp of the natal teeth. This was compared with the stem cell potential of deciduous teeth. Natal teeth exhibited higher immunohistochemical expression, which suggests that discarded tissues like natal teeth are a potential source for stem cells.

First draft submitted: 5 June 2018; Accepted for publication: 15 August 2018; Published online: 4 October 2018

Keywords: CD44 • dental pulp stem cell (DPSC) • desmin • immunohistochemical expression • natal teeth $\bullet$ nestin - Oct-4 • osteopontin and myogenin • SOX2

The first tooth erupts in the human oral cavity at 6 months of age. However, an occasional entity termed 'natal teeth' may be present in some babies at birth. When these teeth erupt within the first month of birth, they are termed neonatal teeth [1]. Although the etiology of early eruption of these teeth is still unknown, the incidence of natal and neonatal teeth ranges between 1:2000 and 1:3500 [2,3]. Although these teeth are not necessarily considered pathological or abnormal, they may lead to several complications including aspiration, swallowing due to premature exfoliation, ulcerations on the tongue and lips as well as injuries to the mother during feeding [4,5]. To avoid the aforementioned complications, these teeth are prophylactically removed.

Dental pulp stem cells (DPSCs) are mainly isolated from the pulp tissue of exfoliated deciduous teeth, deciduous incisors or permanent third molars [6,7]. There have been case reports to show that supernumerary teeth and natal teeth may serve as potential sources of DPSCs [8-10]. Despite being uncommon, natal teeth are seen just after the developmental stage, where the stem cells are in the peak of their activity. Therefore, these teeth may be explored as a source of stem cells, similar to the embryonic sac and the embryonic fluid. However, the stem cell and differentiation marker expression profile of natal cells, in comparison with deciduous teeth, is unknown. The 


\begin{tabular}{|c|c|c|c|}
\hline Primary serum & Clone & Source & Working titer \\
\hline OCT4 & ab18976 & Abcam Cambridge, MA, USA & $1: 100$ \\
\hline SOX2 & Ab97959 & Abcam Cambridge, MA, USA & $1: 100$ \\
\hline CD44 (HCAM) & $A b-4$ & Thermo Fisher Scientific, Fermount, CA, USA & $1: 150$ \\
\hline Nestin & ab93666 & Abcam Cambridge, MA, USA & $1: 120$ \\
\hline Osteopontin & $\mathrm{AKm} 2 \mathrm{~A} 1$ & Santa Cruz Biotechnology Inc., Dallas, TX, USA & $1: 50$ \\
\hline Desmin & D33 & Thermo Fisher Scientific, Fermount, CA, USA & Prediluted \\
\hline Myogenin & F5D & Thermo Fisher Scientific, Fermount, CA, USA & Prediluted \\
\hline CD34 & C-18 & Santa Cruz Biotechnology Inc, Dallas, TX, USA & $1: 150$ \\
\hline
\end{tabular}

aim of this study was to investigate the expression of stem cell/progenitor cell and differentiation markers of natal teeth pulp tissue, compared with pulp tissue obtained from a deciduous tooth, using immunohistochemistry.

\section{Materials \& methods}

Two natal teeth of a 20-day-old healthy male patient and a deciduous mandibular first molar of an 11-year-old healthy male patient were extracted under local anesthesia based on a protocol approved by the Institutional Review Board and Ethics Committee. Informed consent was obtained from the parents of these patients to extract the teeth and use them for further analysis. The natal teeth were extracted for prophylactic reasons as outlined previously, while the deciduous teeth were extracted due to mobility. The deciduous molar served as the positive control. Following extraction, the teeth were immediately fixed in $10 \%$ neutral buffered formalin for $24 \mathrm{~h}$. The dental pulp tissue was removed from the deciduous mandibular first molar by splitting the tooth with a chisel and immediately fixing in $10 \%$ neutral buffered formalin for $24 \mathrm{~h}$.

\section{Histologic procedures}

After fixation, the natal teeth were demineralized in $10 \%$ EDTA $(\mathrm{pH}=7.4)$ for 6 months at room temperature. Subsequently, the samples were rinsed under running water for $4 \mathrm{~h}$ followed by dehydration with ascending concentrations of ethanol. Then, the teeth were deparaffinized in xylene, infiltrated, and embedded in paraffin. With the microtome set at $5 \mu \mathrm{m}$, longitudinal serial sections were cut and stained with hematoxylin-eosin.

\section{Immunohistochemical procedures}

Serial sections, $5 \mu \mathrm{m}$ in thickness, prepared from archived formalin-fixed, paraffin-embedded (FFPE) tissue blocks, were used for immunohistochemical analysis. Sections were deparaffinized and rehydrated following standard methods. Briefly, the sections were deparaffinized three-times with xylene for $5 \mathrm{~min}$ and rehydrated in graded ethanol (80-100\%) for $5 \mathrm{~min}$. Antigen retrieval was performed by boiling at $98^{\circ} \mathrm{C}$ for $40 \mathrm{~min}$ in $0.01 \mathrm{~mol} / \mathrm{l}$ sodium citrate buffer ( $\mathrm{pH}$ 6.0). Details of the primary antibody clones, sources and titers are listed in Table 1.

The endogenous peroxidase activity was blocked and the tissue samples were incubated with OCT4, SOX2, CD44, nestin, osteopontin, desmin and myogenin antibodies for $90 \mathrm{~min}$. Subsequently, the slices were rinsed and incubated with the biotinylated secondary antibody at room temperature for $30 \mathrm{~min}$. The bound antibody complexes were stained for 3-5 min or until appropriate for microscopic examination and then counter stained with hematoxylin (30 s) and mounted.

The expression of OCT4, SOX2, CD44, nestin, osteopontin, desmin, myogenin and CD34 on the natal as well as the deciduous tooth (positive control) tissue samples were analyzed. The immunoreactivity of the specimens was interpreted based on the intensity of the staining. Score ranks usually lie in a range from 'negative' (mostly marked as '-') to 'positive', which was signed with different amount of ' + ' depending upon the intensity of the stain. The samples were examined with conventional light microscope. For OCT4, SOX2, osteopontin, myogenin nuclear immunohistochemical staining was considered positive whereas for nestin and desmin cytoplasmic immunohistochemical staining was considered positive. Membranous immunohistochemical staining was considered positive for CD34 and CD44. The staining intensity was graded as $(+)$ weak, $(++)$ moderate and $(+++)$ intense according to the overall appearance at different powers of magnification, in other words, $4 \times, 10 \times$ and $40 \times$, respectively and (-) for no staining. 
Figure 1. Photomicrograph of the natal tooth. Figure shows: (A) Hematoxylin and eosin-stained section [10x magnification]; (B) Strong immunohistochemical expression of Nestin, restricted to odontoblasts $(40 \times$ magnification); (C) Strong immunohistochemical expression of CD44, restricted to odontoblasts and pulp tissue (40× magnification); (D) Strong expression of SOX2 in pulp tissue ( $40 \times$ magnification).
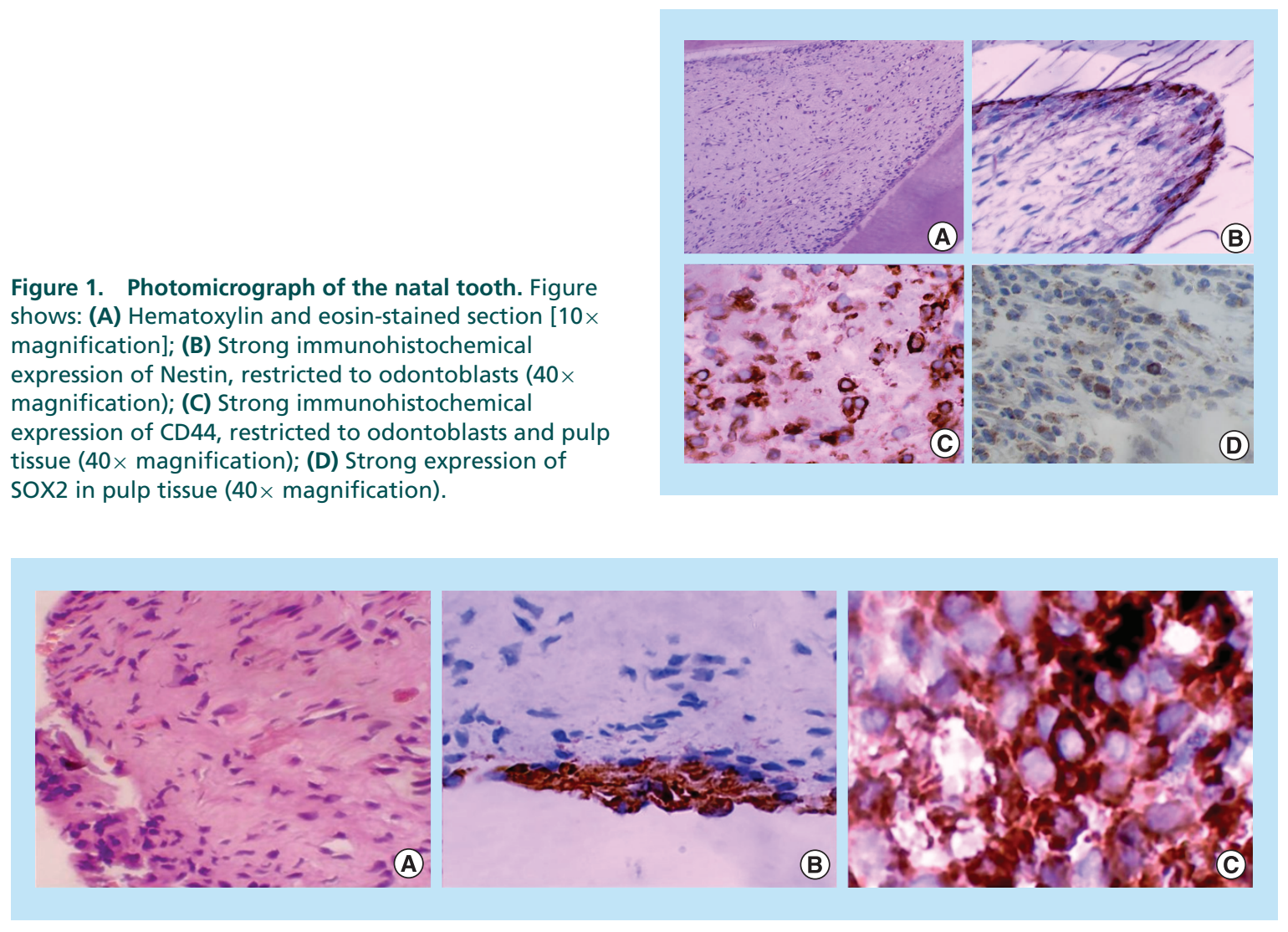

Figure 2. Photomicrograph of the deciduous tooth (control). (A) Hematoxylin and eosin-stained section (40X magnification); (B) Immunohistochemical expression of CD44 in peripheral odontoblasts (40× magnification); (C) Immunohistochemical expression of nestin, restricted to the peripheral odontoblasts (40x magnification).

\begin{tabular}{|c|c|c|c|}
\hline Markers & N1 & N2 & D (control) \\
\hline OCT4 & $\dagger$ & $\dagger$ & $\dagger$ \\
\hline sox2 & $\ddagger$ & $\ddagger$ & $\dagger$ \\
\hline CD44 & $\S$ & $\ddagger$ & $\ddagger$ \\
\hline Nestin & $\S$ & $\S$ & $\ddagger$ \\
\hline Osteopontin & $\dagger$ & $\dagger$ & $\dagger$ \\
\hline Desmin & $\dagger$ & $\dagger$ & $\dagger$ \\
\hline Myogenin & $\dagger$ & $\dagger$ & $\dagger$ \\
\hline CD34 & $\dagger$ & $\dagger$ & $\dagger$ \\
\hline \multicolumn{4}{|c|}{$\begin{array}{l}\text { †No immunostaining. } \\
\text { ¥Moderate. } \\
\text { \& Intense. } \\
\text { D: Deciduous molar; N: Natal tooth. }\end{array}$} \\
\hline
\end{tabular}

\section{Results}

Histological \& immunohistochemical observations

Hematoxylin and eosin stained sections of the tissues obtained from the natal and deciduous teeth showed myxomatous connective tissue with blood vessels and inflammatory cells. A clear odontoblastic lining was evident adjacent to the secondary dentin (Figures $1 \mathrm{~A} \& 2 \mathrm{~A}$ ).

Immunohistochemical examination (Table 2) demonstrated that both the natal and deciduous teeth expressed nestin and CD44 immunopositivity for odontoblasts and connective tissue. However, expression of all the markers was stronger in the natal teeth than the deciduous tooth. In contrast, SOX2 was expressed only by natal teeth. 
Remaining immunohistochemical markers such as osteopontin, desmin, myogenin, OCT4 and CD34 were negative in both the teeth.

\section{Discussion}

Stem cells are an excellent source of generation of a huge number of mature cells through a sequential procedure of proliferation and differentiation, retaining their ability to self renew to maintain the stem cell pool. Stem cell markers are the protein products that identify a multipotent single cell capable of recapitulating. There are four types of cell: embryonic stem cell (ESC), hematopoietic stem cell (HSC), mesenchymal/stromal stem cell (MSC/SSC) and neural stem cell (NSC).

The purpose of this study was to characterize the stem cellness of the dental pulp obtained from natal teeth, which are in general considered waste teeth, using histological and immunohistochemical methods. Only two studies thus far have successfully isolated and characterized human natal DPSCs (hNDPSCs) in vitro [9,10]. Although Karaoz et al. [9] demonstrated the expression of ESC markers (OCT4, REX1, FOXD3, SOX2 and Nanog) and differentiation potential of DPSCs derived from natal teeth toward adipogenic, chondrogenic, osteogenic, myogenic and neurogenic lineages, Suchanek et al. [10] demonstrated successful in vitro differentiation of hNDPSCs into induced pluripotent cells that were morphologically similar to pancreatic islet cells.

Prior to investigating the multilineage differentiation potential of DPSC, an overview of marker expression in basic pulp tissue is crucial. In the present study, for the first time, we demonstrate the immunohistochemical characterization of stem cell and differentiation markers in FFPE tissue sections of natal teeth. OCT4 and SOX2 serve as reliable markers for identifying the NSC population. OCT4 is an ESC marker, identified as a DNA-binding protein that activates gene transcription via an octamer motif [11]. OCT4 expression is required to sustain stem cell self-renewing capacity and pluripotency. SRY-box2, also known as SOX2 is a transcription factor, known to be expressed at high levels in the neuroepithelium of the developing CNS, and is considered to be critical for NSC proliferation and differentiation [12]. Previous studies have identified that the stem cell or potential progenitor cell population in the dental pulp constitutes $<1 \%$ of the total cells [13,14]. This may be attributed to the nonexpression of stem cell markers (OCT4 and SOX2) in the deciduous pulp (positive control in this study). The present study showed positive immune expression of SOX2 in the dental pulp of natal teeth, which confirms the presence of higher percentage of stem/progenitor cell population compared with the deciduous pulp (Figure 1D).

Expression of CD44, a positive MSC marker and CD34, a negative MSC marker were also analyzed in this work. CD44, a ubiquitous, multistructural, multifunctional cell surface glycoprotein is involved in cell-to-cell adhesion, cell-matrix interactions and cell migration. It is widely expressed in several cell types. CD44 is expressed at the time of extracellular matrix formation in many sites during embryonic development. CD44, a cell-adhesion factor, is involved in the induction of mineralization in the dental pulp. Interestingly, it was overexpressed in the natal tooth specimen when compared with the control (Figures 1C \& 2B). CD34 is an HSC marker expressed on a small fraction of human bone marrow cells. Since the CD34 positive enriched cell population from the bone marrow is responsible for most of its hematopoietic activity, it is considered to be one of the most critical markers of HSCs. This study showed immunonegativity of CD34 in the natal as well as the control deciduous molar, thus corroborating with the findings of Karaoz et al. [9].

Nestin, a class VI intermediate filament protein, is predominantly expressed in the CNS [15,16]. Although nestin does not form intermediate filaments by itself, it co-assembles with vimentin or alpha-internexin to form a heterodimer coiled-complex, which are intermediate filaments. Its transient expression has been suggested to be a major step in neural differentiation. However, it is also expressed in non-NSC populations such as pancreatic islet progenitors and hematopoietic progenitors and DPSCs. In young developing human deciduous tooth germs, nestin immunoreactivity has been reported in the odontoblast, pulp fibroblasts, odontoblastic processes up to the dentinoenamel junction and pulp cells. In mature teeth, expression of nestin appears to be restricted to odontoblasts. The findings of the present study are in agreement with previous findings (Figures 1B \& 2C) [17].

Natal teeth provide a unique population of stem cells that can be attributed to their entirely intrauterine development. They are different from deciduous and permanent teeth in their structure as well as function. In their study on exfoliated deciduous teeth, Miura et al. demonstrated a significant difference between SHED and DPSCs in terms of rates of proliferation, cell population doublings, sphere-like cell cluster formation and osteoinductive capacity, all of which were better in SHED. Our study, which compared natal teeth and deciduous teeth, also found these teeth as an excellent and abundant source of stem cells. 
An interesting finding in the present study that differed to the results of Karaoz et al. is the lack of expression of myogenic (desmin and myogenin) and osteogenic differentiation markers (osteopontin). This may be attributed to the fact that the above-mentioned study used cell culture techniques, in contrast to our report, which used FFPE tissues. Our study provides evidence that natal teeth have shown very strong positive expression of CD44 (MSC), SOX2 (ESC) and Nestin (NSC) markers. Natal teeth therefore may be a viable source of stem cells to induce repair or regeneration of tissue, wherever applicable and for treatment of degenerative tissues. To the best of our knowledge, this is the first study to demonstrate positive immunoexpression of various stem cell markers in FFPE sections of a usually discarded tooth such as the natal tooth.

\section{Conclusion}

Taken together, the results of this study support the use of natal teeth as a potential source of pluripotent stem cells for future cell-based therapies.

\section{Future perspective}

Since the natal teeth develop during the intrauterine stages, the stem cells of natal teeth originate at much earlier stages of development and exhibit excellent potency to differentiate into various structures. This property could be utilized for treating various clinical conditions. They will also be useful in dentin tissue engineering, teeth replantation and pulp revascularization. However, the stem cells of natal teeth origin have been heretofore underestimated due to the sparse available literature and hence future research in this direction is warranted to use them to their maximal potential.

\section{Summary points}

- The immunohistochemical expression of OCT4, SOX2, CD34, CD44, nestin, osteopontin, desmin, myogenin and KI67 on the dental pulp of natal as well as the deciduous tooth tissue samples was analyzed.

- The natal teeth as well as the deciduous tooth specimens expressed immunopositivity for nestin and CD44. However, the natal teeth specimen showed an overexpression of these markers compared with its counterpart.

- SOX2, a transcription factor considered to be critical for neural stem cell proliferation and differentiation, was expressed only by the natal teeth.

- Natal teeth, a usually discarded tissue, can be considered as a potential source of pluripotent stem cells for future cell-based therapies.

\section{Authors' contributions}

H Shetty designed the study and performed the experiments; A Kakade performed the clinical procedures: S Shetty analyzed the data. P Neelkantan analyzed the data and wrote the manuscript; S Nagar interpreted the results; R Desai critically reviewed the manuscript; K Beri designed the study and edited the manuscript for language quality.

Financial \& competing interests disclosure

The authors have no relevant affiliations or financial involvement with any organization or entity with a financial interest in or financial conflict with the subject matter or materials discussed in the manuscript. This includes employment, consultancies, honoraria, stock ownership or options, expert testimony, grants or patents received or pending, or royalties.

No writing assistance was utilized in the production of this manuscript.

\section{Ethical conduct of research}

The authors state that they have obtained appropriate institutional review board approval or have followed the principles outlined in the Declaration of Helsinki for all human or animal experimental investigations. In addition, for investigations involving human subjects, informed consent has been obtained from the participants involved.

\section{Open access}

This work is licensed under the Creative Commons Attribution 4.0 License. To view a copy of this license, visit http://creativecomm ons.org/licenses/by/4.0/ 


\section{References}

Papers of special note have been highlighted as: $\bullet$ of interest

1. Massler M, Savara BS. Natal and neonatal teeth: a review of 24 cases reported in the literature. J. Pediatr. 36(3), 349-359 (1950).

- Provides an overview of natal and neonatal teeth and a better understanding of the etiology and management of those teeth.

2. El. Khatib K, Abouchadi A, Nassih M et al. Natal teeth: apropos of five cases. Rev. Stomatol. Chir. Maxillofac. 106(6), 325-327 (2005).

3. Dyment H, Anderson R, Humphrey J, Chase I. Residual neonatal teeth: a case report. J. Can. Dent. Assoc. 71(6), 394-397 (2005).

4. Zhu J, King D. Natal and neonatal teeth. ASDC J. Dent. Child. 62, 123-128 (1995).

5. Sothinathan R., Shakib K. Natal teeth: a sign of fortuity or grave misfortune. Br. Dent. J. 6, 265-266 (2011).

6. Gronthos S, Mankani M, Brahim J, Robey PG, Shi S. Postnatal human dental pulp stem cells (DPSCs) in vitro and in vivo. Proc. Natl Acad. Sci. USA 97(25), 13625-13630 (2000).

- Helps us to understand that dental pulp cells have the ability to form dentin/pulp-like complex.

7. Miura M, Gronthos S, Zhao M et al. SHED: stem cells from human exfoliated deciduous teeth. Proc. Natl Acad. Sci. USA 100(10), 5807-5812 (2003).

- Focuses on exfoliated human deciduous teeth as a source of multipotent stem cells.

8. Huang AH, Chen YK, Lin LM, Shieh TY, Chan AW. Isolation and characterization of dental pulp stem cells from a supernumerary tooth. J. Oral. Pathol. Med. 37(9), 571-574 (2008).

- Demonstrates the feasibility of deriving dental pulp stem cells (DPSCs) from other waste tissues like mesiodens, thus reinforcing the novel approach of using discarded tissue, such as natal teeth.

9. Karaöz E, Doğan BN, Aksoy A et al. Isolation and in vitro characterization of dental pulp stem cells from natal teeth. Histochem. Cell Biol. 133(1), 95-111 (2010).

- Helps us to understand the potential of natal teeth as a source of stem cells by isolating and extensively characterizing the stem cells of these teeth.

10. Suchánek J, Nasry SA, Soukup T. The differentiation potential of human natal dental pulp. Stem cells into insulin-producing cells. Folia Biol (Praha). 63(4), 132-138 (2017).

- Establishes the potential of DPSCs obtained from neonatal teeth to differentiate into insulin producing cells and their potential use in stem cell therapy of diabetes mellitus.

11. Scholar H, Rupert S, Suzuki N, Chowdary K, Gruss P. New type of POU domain in germ line-specific protein in Oct-4. Nature 344, 435-439 (1990).

12. Liu P, Cai J, Dong D et al. Effects of SOX2 on proliferation, migration and adhesion of human dental pulp stem cells. PLoS ONE 10(10), e0141346 (2015).

13. Gronthos S, Brahim J, Li W et al. Stem cell properties of human dental pulp stem cells. J. Dent. Res. 81(8), 531-535 (2002).

- Describes properties of DPSCs like self-renewal, multilineage differentiation capacity, clonogenic efficiency, and so forth, and provides evidence to show that DPSCs belong to a novel population of postnatal stem cells.

14. Waddington RJ, Youde SJ, Lee CP, Sloan AJ. Isolation of distinct progenitor stem cell populations from dental pulp. Cells Tissues Organs 189(1-4), 268-274 (2009).

15. Dhalstrand J, Collins VP, Lendhahl U. Expression of the class VI intermediate filament nestin in human central nervous system tumors. Cancer Res. 52(19), 5334-5341 (1992).

16. Lendahl U, Zimmerman LB, McKay RDG. CNS stem cells express a new class of intermediate filament protein. Cell 60(4), 585-595 (1990).

17. About I, Maquin DL, Lendahl U, Misiadis TA. Nestin expression in embryonic and adult human teeth under normal and pathological conditions. Am. J. Pathol. 157(1), 287-295 (2000).

- Provides a better understanding of nestin expression in pulp of developing and mature human teeth. 